\title{
Recognition of DHN-melanin by the C-type lectin, MelLec, is required for protective immunity to Aspergillus fumigatus
}

Mark H.T. Stappers, Alexandra E. Clark, Vishukumar Aimanianda, Stefan Bidula, Delyth M. Reid, Patawee Asamaphan, Sarah E. Hardison, Ivy M. Dambuza, Isabel Valsecchi, Bernhard Kerscher, Anthony Plato, Carol A. Wallace, Raif Yuecel, Betty Hebecker, Maria da Glória Teixeira Sousa, Cristina Cunha, Yan Liu, Ten Feizi, Axel A. Brakhage, Kyung J. Kwon-Chung, Neil A. R. Gow, Matteo Zanda, Monica Piras, Chiara Zanato, Martin Jaeger, Mihai G. Netea, Frank L. van de Veerdonk, João F. Lacerda, António Campos Jr., Agostinho Carvalho, Janet A. Willment, Jean-Paul Latgé and Gordon D. Brown.

\section{Summary}

C-type lectin receptors play a crucial role in recognition of fungi and induction of protective antifungal immune responses.

Here, we identify a C-type lectin receptor, Melanin sensing C-type Lectin receptor (MelLec), which is essential for protective immunity to Aspergillus fumigatus.

MelLec:

Recognizes fungal DHN-melanin.

Is expressed on CD31+endothelial cells in various murine tissues.

Plays a crucial role in controlling systemic $A$. fumigatus infection in a murine model.

Polymorphism Gly26Ala, when present in donor cells, increases the susceptibility of stem-cell transplant patients to disseminated aspergillosis.

Published in: Stappers et al. Nature, 2018 Mar 15;555(7696):382-386.

\section{MelLec recognizes DHN-melanin in conidial spores of Aspergillus fumigatus}

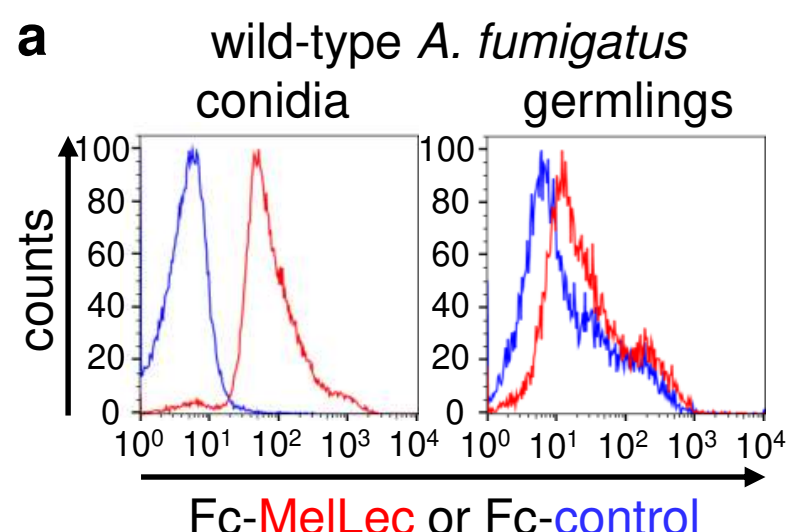

Fc-MelLec or Fc-control

A. fumigatus
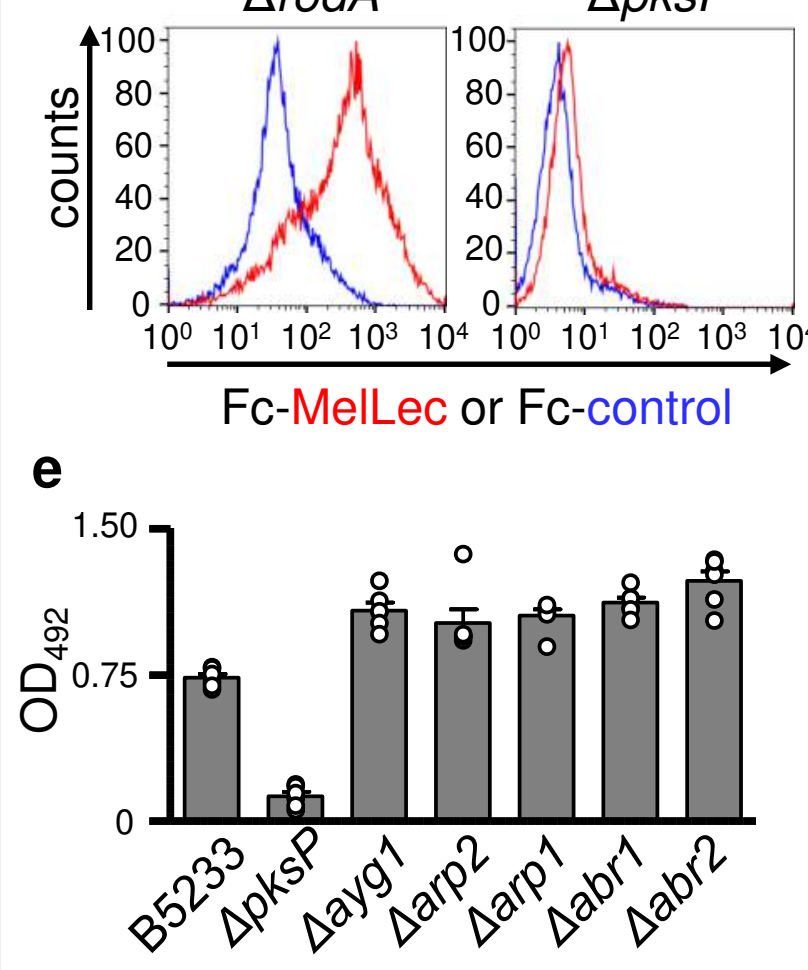

b

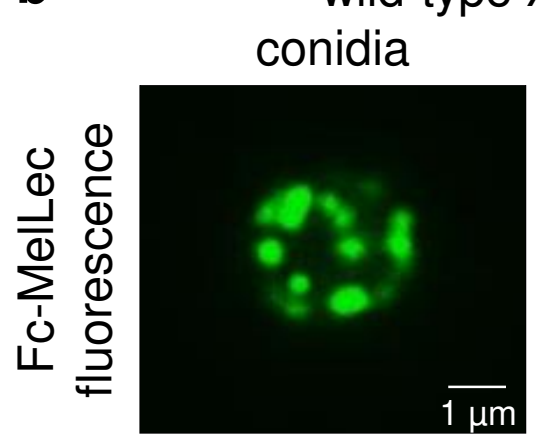

d
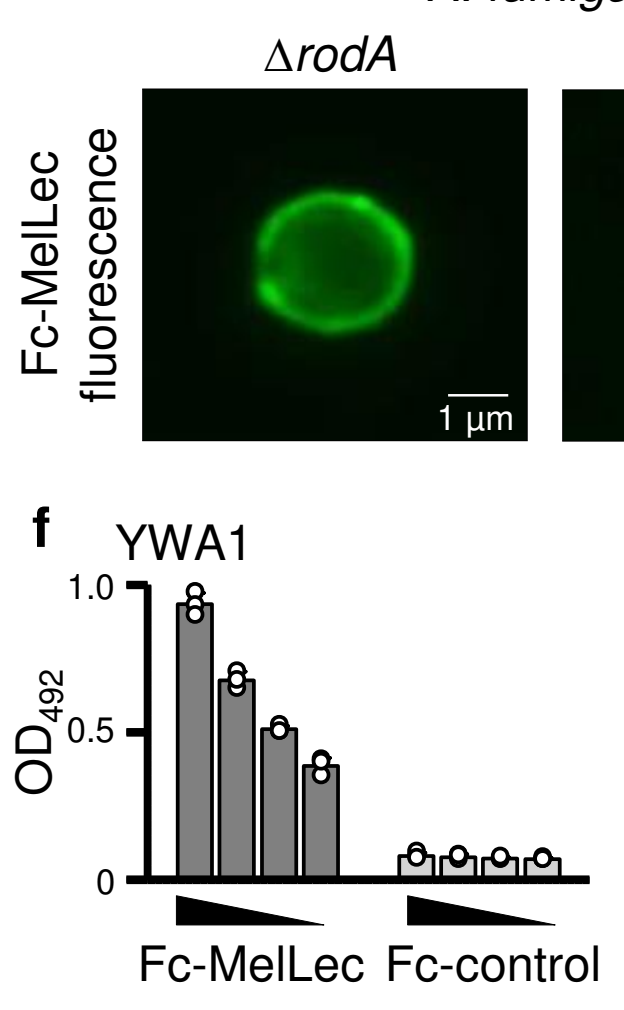

Figure 1. Histograms (a) and immunofluorescence micrographs (b) using Fc-MelLec to detect ligands on wild-type A. fumigatus conidia and following conidial swelling and germination over time. Histograms (c) and immunofluorescence micrographs (d) using Fc-MelLec to detect ligands on rodlet-deficient, melanin-containing $(\triangle \operatorname{rod} A)$ or melanindeficient $(\triangle p k s P) \quad A$. fumigatus conidia, as indicated. Detection of MelLec ligands on $A$. fumigatus $\mathrm{DHN}$-melanin synthesis pathway mutants (e), and DHN-melanin intermediate YWA1 (f) by ELISA.

\section{Acknowledgements}

\section{MelLec is expressed on endothelial cells}
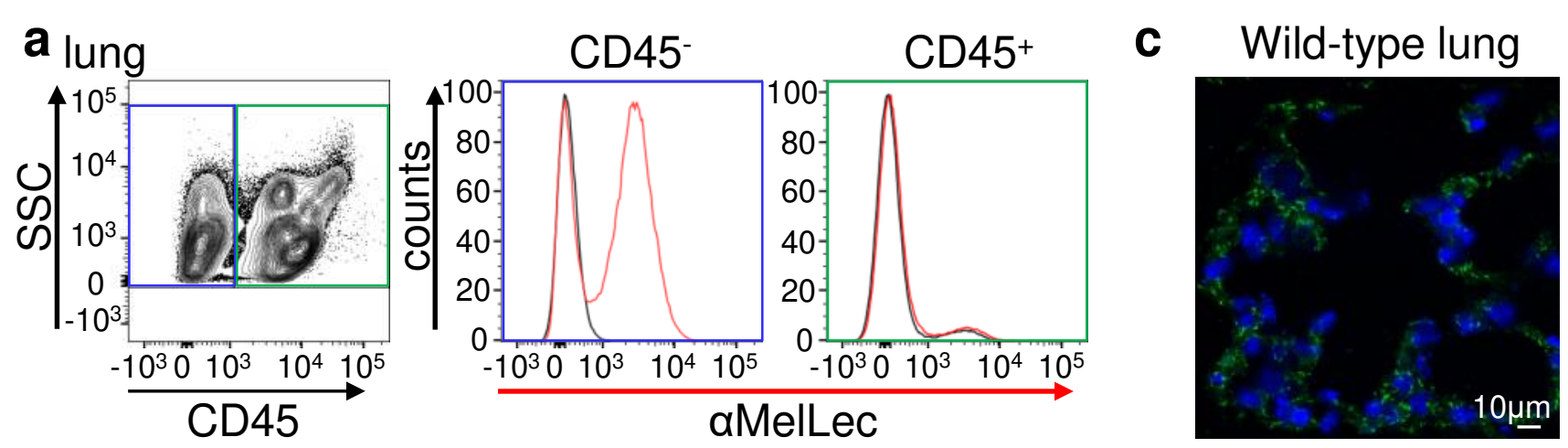

b lung CD45-
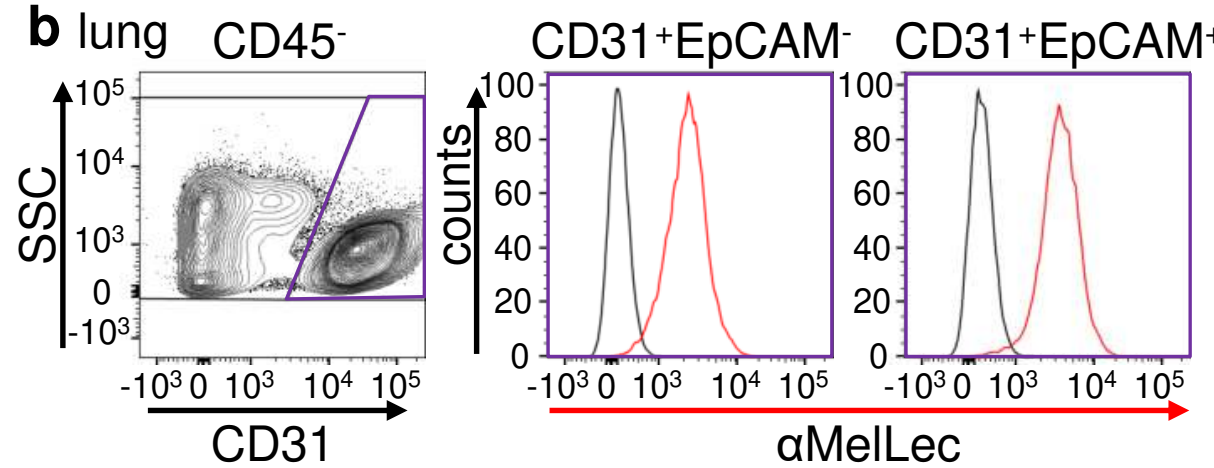

MelLec $^{-/-}$lung

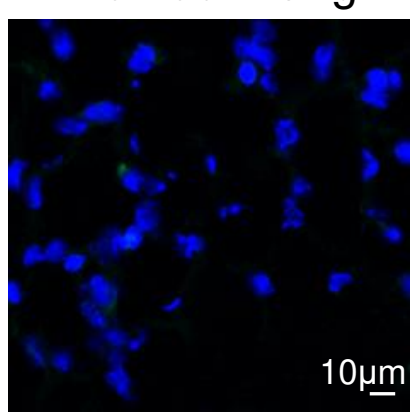

Figure 2. a-b, Flow cytometric analysis of MelLec expression on live cell populations in the murine lung, as indicated. c, Immunofluorescence microscopy of naïve lung tissue from wild-type and MelLec-/- mice stained with aMelLec (green).

\section{MelLec-/- mice display increased} susceptibility to invasive aspergillosis
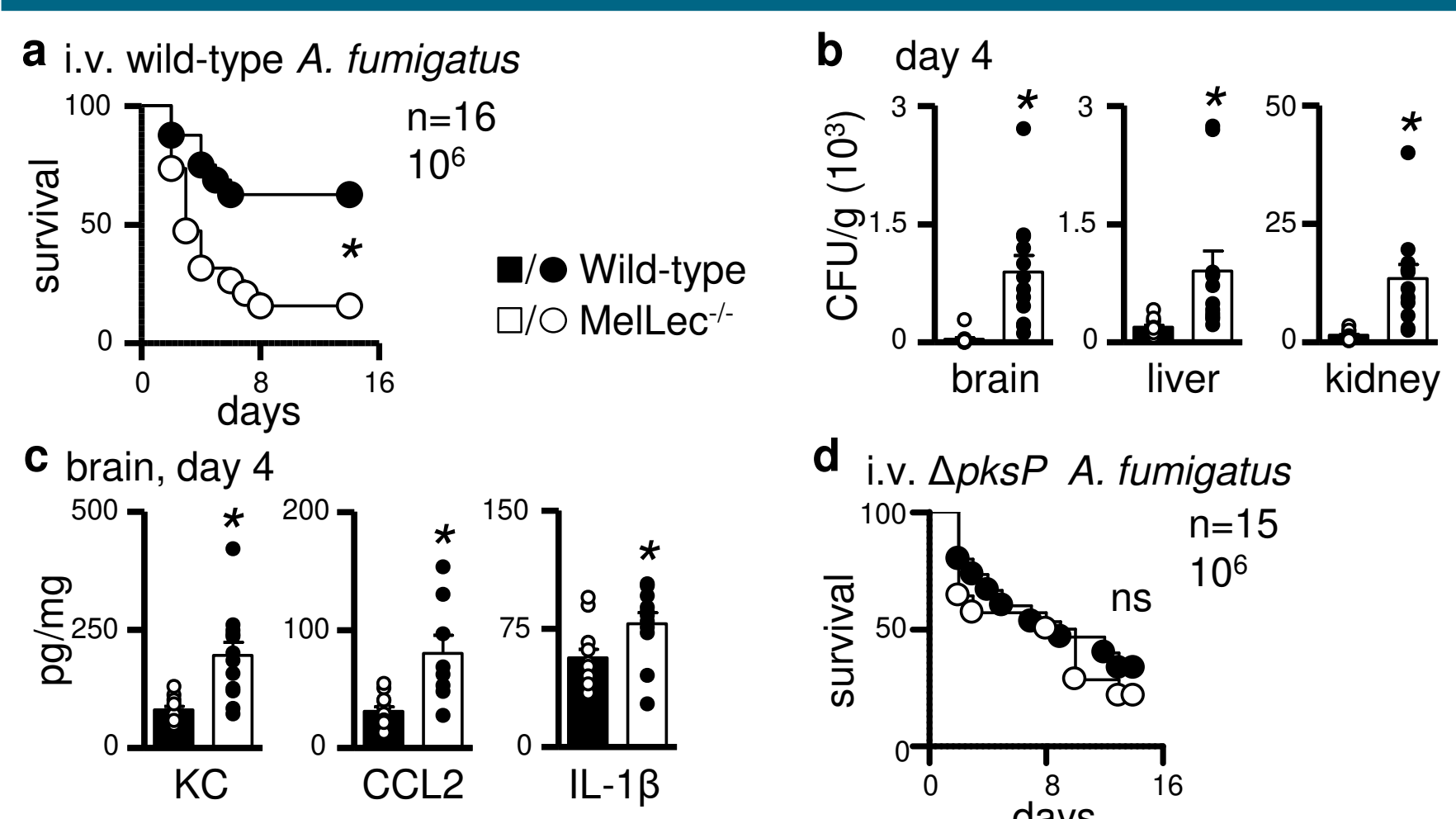

d i.v. $\Delta p k s P$ A. fumigatus

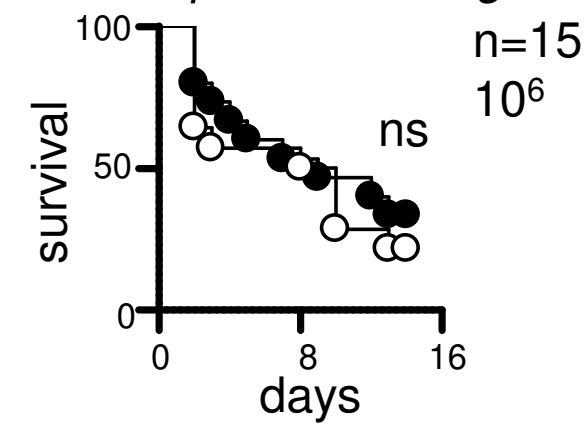

Figure 3. a, Survival of mice following intravenous (i.v.) infection with $10^{6}$ A. fumigatus wild-type conidia. Tissue fungal burdens (b) and brain cytokine levels (c) in mice 4 days after i.v. infection with $10^{6} A$. fumigatus conidia. d, Survival of mice following i.v. infection with $10^{6}$ melanin-deficient $(\triangle p k s P) A$. fumigatus conidia.

\section{MelLec SNP increases risk of aspergillosis}

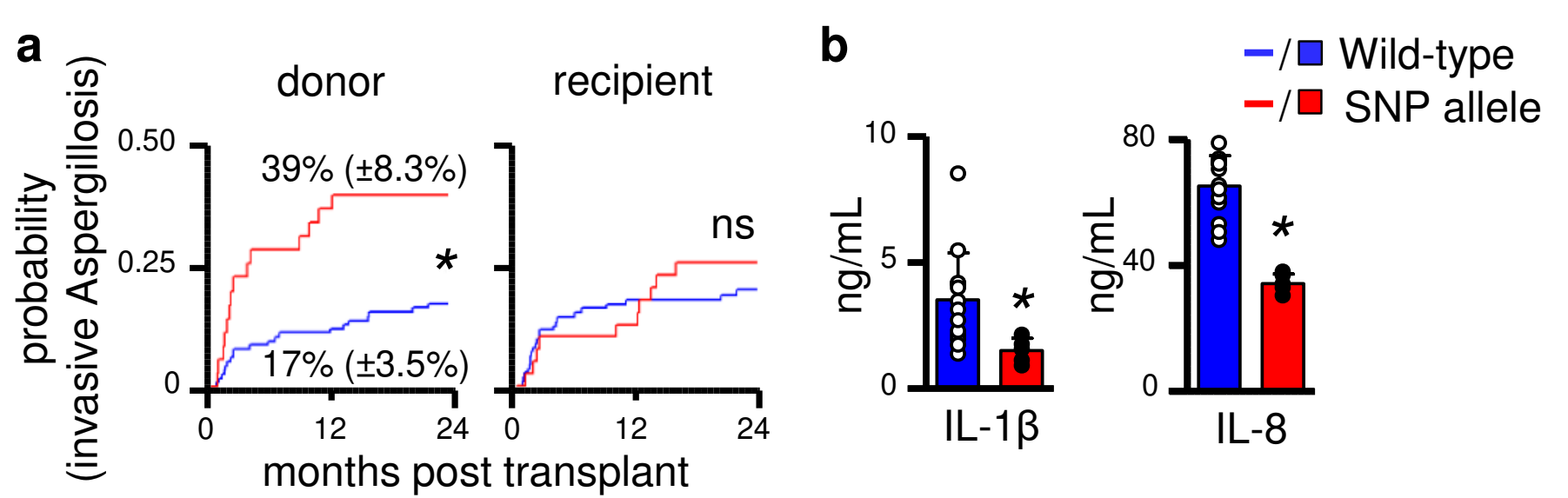

Figure 4. a, Cumulative incidence analysis of invasive aspergillosis after stem-cell transplantation according to donor (wild-type, $\mathrm{n}=238$; SNP allele, $\mathrm{n}=72$ ) or recipient (wild-type, $\mathrm{n}=228$; SNP allele, $\mathrm{n}=80$ ) CLEC1A rs2306894 genotypes. b. Inflammatory cytokine production in monocyte-derived macrophages, following stimulation with $A$. fumigatus conidia (wild-type, $n=14$; SNP allele, $n=5$ ). 